\title{
Cuidados de Enfermagem para detecção de violência contra idosos
}

\author{
Nursing Care for the detection of violence against the elderly \\ Cuidados de enfermería para detección de violencia contra ancianos
}

\section{Caterine Oliveira Azevedo ${ }^{1 *}$, Thiago Augusto Soares Monteiro da Silva ${ }^{2}$ \\ Como citar esse artigo. Azevedo, \\ CO; da Silva, TASM. Cuidados de

\author{
Resumo
}

Enfermagem para detecção de violência contra idosos. Revista Pró-UniverSUS. 2019 Jan./Jun.; 10 (1): 55-59.

Trata-se de um estudo exploratório de caráter descritivo com abordagem qualitativa realizado com 20 enfermeiros de um Hospital Universitário da Região Centro-Sul Fluminense do Estado do Rio de Janeiro. Foi utilizado um questionário semiestruturado com perguntas abertas e fechadas. A estratégia analítica usada para analisar os dados foi a análise de conteúdo. Os dados foram analisados e discutidos levando-se em consideração o conteúdo teórico apresentado na revisão de literatura. Constatou-se que os enfermeiros têm conhecimento abrangente sobre o conceito de violência, que ao identificarem situação de violência contra a pessoa idosa, eles se valem de diversas estratégias de proteção ao idoso e, consequentemente de enfrentamento da violência. Porém ficou evidente que diversos fatores intrínsecos ao idoso contribuem para dificultar a identificação da violência pelos enfermeiros, como medo, vergonha e omissão por medo do agressor ou por não querer denunciá-los por questões de afinidades parentais. Quanto ao reconhecimento dos sinais físicas evidentes, considera-se que este demande muita cautela e conhecimento por parte dos enfermeiros para não confundir esses sinais com sinais decorrentes do próprio envelhecimento, com suposta violência. Diante da fragilidade da situação, o enfermeiro precisar ter além do conhecimento técnico-científico, sensibilidade para abordar adequadamente o idoso, de forma que consiga colher dele a maior quantidade possível de informações sobre a violência sofrida.

Palavras-chave: Assistência de Enfermagem; Enfermagem Forense Violência; Idoso.

\begin{abstract}
This is an exploratory descriptive study with a qualitative approach performed with 20 nurses from a University Hospital in the Center-South Fluminense Region of the State of Rio de Janeiro. A semi-structured questionnaire was used with open and closed questions. The analytical strategy used to analyze the data was content analysis. The data were analyzed and discussed taking into account the theoretical content presented in the literature review. It was verified that the nurses have a comprehensive knowledge about the concept of violence, that in identifying a situation of violence against the elderly, they use different strategies to protect the elderly and, consequently, to confront violence. However, it was evident that several factors intrinsic to the elderly contribute to make it difficult to identify the violence by nurses, such as fear, shame and omission for fear of the aggressor or for not wanting to denounce them for reasons of parental affinities. Regarding the recognition of the obvious physical signs, it is considered that this requires a lot of caution and knowledge on the part of the nurses not to confuse these signs with signs of aging itself, with alleged violence. Faced with the fragility of the situation, the nurse needs to have, besides the technical-scientific knowledge, the sensitivity to adequately address the elderly, so that he can obtain as much information about the violence as possible.
\end{abstract}

Keywords: Nursing care; Forensic Nursing; Violence; Old man.

\section{Resumen}

Se trata de un estudio exploratorio de carácter descriptivo con abordaje cualitativo realizado con 20 enfermeros de un Hospital Universitario de la Región Centro-Sur Fluminense del Estado de Río de Janeiro. Se utilizó un cuestionario semiestructurado con preguntas abiertas y cerradas. La estrategia analítica utilizada para analizar los datos fue el análisis de contenido. Los datos fueron analizados y discutidos tomando en consideración el contenido teórico presentado en la revisión de literatura. Se constató que los enfermeros tienen conocimiento integral sobre el concepto de violencia, que al identificar situación de violencia contra la persona anciana, ellos se valen de diversas estrategias de protección al anciano y, consecuentemente de enfrentamiento de la violencia. Pero quedó evidente que diversos factores intrínsecos al anciano contribuyen a dificultar la identificación de la violencia por los enfermeros, como miedo, vergüenza y omisión por miedo al agresor o por no querer denunciarlos por cuestiones de afinidades parentales. En cuanto al reconocimiento de los signos físicos evidentes, se considera que esta demanda mucha cautela y conocimiento por parte de los enfermeros para no confundir esos signos con señales derivadas del propio envejecimiento, con supuesta violencia. Ante la fragilidad de la situación, el enfermero necesita tener más allá del conocimiento técnico-científico, sensibilidad para abordar adecuadamente al anciano, de forma que consiga recoger de él la mayor cantidad posible de informaciones sobre la violencia sufrida.

Palabras clave: Asistencia de enfermería; Enfermería Forense; la violencia; personas de edad avanzada.

Afiliação dos autores:

1Acadêmico do Curso de Enfermagem, Universidade de Vassouras. RJ, Brasil. Email: cattyoazevedo@yahoo.com.br ORCID: https://orcid.org/0000-0001-6822-6645

2Mestre. Professor do Curso de Enfermagem, Universidade de Vassouras. RJ, Brasil. Email: thiagoams@bol.com.br ORCID: https://orcid.org/0000-0001-6870-5101 


\section{Introdução}

O envelhecimento acelerado da população mundial tem se tornado um grande desafio do século XXI. $\mathrm{O}$ aumento da expectativa de vida e, consequentemente, o maior número de idosos entre a população em geral, é uma realidade vivenciada pela maioria dos países, incluindo o Brasil. Tal fato vem indicando a necessidade de mudanças nas políticas sociais e novos desafios para a saúde pública. ${ }^{1}$

Entre os problemas sociais decorrentes do rápido envelhecimento populacional destaca-se o aumento da violência contra a pessoa idosa. A violência contra a pessoa idosa é considerada um problema social grave, um fenômeno universal que acomete todas as classes sociais, independente de status socioeconômico, etnia e religião. Nas últimas décadas, vem se destacando como um problema de saúde pública, causando adoecimento físico, psíquicos e podendo culminar com a morte. ${ }^{2}$

No Brasil, informações sobre doenças, lesões e traumas de causas violentas em idosos são pouco consistentes, uma vez que os casos de agressões não explicitas, como os casos não discriminados que acontecem no ambiente intrafamiliar, são bastante complexos, delicados e geralmente silenciados, "por envolverem relações e sentimento de insegurança, medo, conflitos de consanguinidade, proximidade, afetividade, relações de amor e instinto de proteção em defesa do agressor." 3:322

A falta de conhecimento dos profissionais de saúde em relação às situações de violência contra a pessoa idosa é outro fator que concorre sobremaneira para a subnotificação, sendo enfatizada por alguns autores. ${ }^{4,3,5}$

Diante do exposto, a problemática desta pesquisa surgiu ao observar que embora existam casos explícitos de violência e maus-tratos contra idosos, muitas vezes esses eventos são imperceptíveis e encobertos, até mesmo pelo próprio idoso vitimado. Assim, pressupõese que o enfermeiro possa encontra dificuldades em detectar os sinais e sintomas de violência e maus tratos contra a pessoa idosa assistida por ele, impedindo-o de ter uma conduta de intervenção adequada.

Objetiva-se verificar quais são as condutas adotadas pelo profissional enfermeiro ao identificar situações de violência e maus-tratos contra a pessoa idosa.

Este estudo se justifica pelo fato da violência contra a pessoa idosa ser uma questão complexa e de difícil resolução, que pode passar despercebida, principalmente em um cenário em que outros problemas clínicos intrínsecos à velhice já se destacam, podendo, contudo, ser um agravante que exige intervenções específicas do profissional de enfermagem.

Contribuir para reforçar o interesse dos enfermeiros em relação à violência contra os idosos, estimulando esses profissionais a concentrar sua atenção na identificação da violência praticada contra essas pessoas. Como fonte de consulta e pesquisa, auxiliando na formação e especialização de recursos humanos e no fornecimento de subsídios para que outras pesquisas sejam realizadas

\section{Metodologia}

Trata-se de um estudo exploratório de caráter descritivo, abordagem qualitativa.

O estudo teve como cenário um Hospital Universitário localizado na Região Centro Sul Fluminense do Estado do Rio de Janeiro.

Participaram do estudo 20 enfermeiros, lotados em diversos setores do Hospital, entre os quais os Setores de Urgência e Emergência, Enfermarias de Clínica Médica e Cirúrgica de adultos, Unidades de Terapia Intensiva e Hemodiálise. Para a realização da coleta de dados foi realizado contato pessoal com os enfermeiros nas referidas unidades, a fim de apresentar os objetivos do estudo e solicitar que os mesmos participassem da pesquisa.

Foram incluídos na pesquisa enfermeiros, de ambos os sexos com pelo menos 2 anos de atuação nos setores. Foram excluídos da pesquisa os enfermeiros que estavam de férias ou licença no período da pesquisa e os técnicos e auxiliares de Enfermagem.

O projeto de pesquisa foi submetido ao Comitê de Ética em Pesquisa da Universidade de Vassouras e aprovado sob o número de Parecer: 2.967.288 e CAAE 96135918.5.0000.5290

A autorização dos enfermeiros foi registrada através da assinatura do Termo de Consentimento Livre e Esclarecido (TCLE) com garantia do sigilo, confidencialidade e anonimato, sendo assim assegurados os aspectos éticos da pesquisa com seres humanos conforme a Resolução 466/12.

A participação na pesquisa não trouxe nenhum tipo de complicação para a saúde dos enfermeiros e nenhuma complicação de fórum legal.

Benefícios do estudo: contribuir para reforçar o interesse dos enfermeiros em relação à violência contra o idoso, estimulando esses profissionais a concentrar sua atenção na identificação da violência praticada contra essas pessoas.

A coleta de dados ocorreu nos meses de outubro e novembro de 2018. Utilizando-se como ferramenta um questionário semiestruturado com 14 perguntas abertas e fechadas, sendo cinco referentes a dados sociodemográficos e nove centradas nos objetivos da 
pesquisa em questão.

A análise dos dados aconteceu a partir da transcrição, leitura do material e a interpretação dos dados, produzidos de acordo com a metodologia e à luz da análise de conteúdo, pelo fato deste método defender a descrição objetiva, sistematizada e qualitativa do conteúdo manifesto das comunicações para interpretação dos dados. ${ }^{6}$

Os dados foram analisados e discutidos levandose em consideração o conteúdo teórico apresentado na revisão de literatura sobre o assunto em questão. O que significa que foram examinados e categorizados em temas e subtemas. Identificação da violência contra o idoso; dificuldades para identificar episódios de violência; condutas adotadas mediante suspeita e/ou confirmação de violência contra o idoso; conhecimento do enfermeiro acerca da Enfermagem Forense.

\section{Resultados e Discussão}

\section{Caracterização dos sujeitos}

No que se refere à idade dos enfermeiros, verificou-se que a idade mínima foi de 29 anos e a máxima de 55 anos. A distribuição por faixa etária permitiu constatar que 45\% (09) dos enfermeiros encontravam-se na faixa compreendida entre 31 a 35 anos, enquanto que 20\% (04) tinham entre 36 a 40 anos, $15 \%(03)$ entre 26 a 30 anos, $5 \%(01)$ entre 41 a 45 anos, $5 \%$ (01) entre 46 a 50 anos, e, finalizando, 5\% (01) entre 51 a 55 anos. Um enfermeiro (5\%) não forneceu essa informação.

Em relação ao sexo verifica-se que a população do estudo foi constituída por maioria masculina, ou seja, 55\%, enquanto que a feminina representou $45 \%$. Quanto ao tempo de formação, observa-se pelos dados, que $45 \%$ (09) dos enfermeiros tinham entre 6 e 10 anos de formados e que 40\% (08) referiram já estar formados por um período compreendido entre 11 e 15 anos, portanto, pelos resultados demonstrados, contata-se que a maioria já tem um longo tempo de formação.

Da mesma forma, ao observar o tempo de atuação dos enfermeiros nos distintos setores, verifica-se que a grande maioria já trabalha um bom tempo em seus setores de atuação, onde $55 \%$ (11) têm entre 6 a 10 anos, enquanto $15 \%$ (03) têm de 2 a 5 anos, 10\% (02) de 11 a 15 anos e outros 20\% (04) têm mais de 15 anos de atuação. Esses resultados demonstram a inexistência de grande rotatividade nas ocupações dos setores do Hospital pela Enfermagem, como também caracteriza um grupo de trabalhadores com bastante experiência e maturidade profissional. 7:649

Verifica-se entre os sujeitos que todos fizeram pelo menos um curso de pós- graduação lato-sensu. Sendo que $50 \%$ (10) dos enfermeiros referem ter feito um curso; 40\% (08) fizeram dois, 5\% (01) três cursos e $5 \%$ (1) quatro cursos. Entre os cursos citados, os mais requisitados foram Cardiologia, Terapia Intensiva, Hemodinâmica, Urgência e Emergência, Neonatologia, Gestão Hospitalar e Nefrologia. Numa análise geral, este fato reflete o perfil de um grupo de profissionais que valoriza a continuidade da própria educação, possivelmente visando um melhor desempenho das suas funções.

\section{Conceito de violência}

Observa-se que existe um consenso sobre o conceito de violência, bem como uma uniformidade em relação à tipologia e aos agravos provocados pela mesma, como se pode observar nos relatos dos enfermeiros (as). A violência é conceituada como uso intencional da força física, verbal, psicológica, sexual com violação dos direitos humanos ou ameaça que resulte em acidente, morte, torturas, conflitos, fome ou trauma psicológico com um indivíduo em sua integridade.

Conforme a Organização Mundial da Saúde, violência é "o uso intencional da força física ou poder, em ameaça ou na prática, contra si próprio, outra pessoa ou contra um grupo ou comunidade, que resulte ou possa resultar em sofrimento, morte, dano psicológico, desenvolvimento prejudicado ou privação". 8:1165 Tratase de um conceito referentes aos processos e às relações interpessoais de grupos, de classes e de gênero, ou objetivadas em instituições, quando se faz uso de diferentes formas, métodos e meios de aniquilamento de outrem, ou de sua coação direta ou indireta, resultando em danos físicos, mentais e morais. ${ }^{9}$

\section{Cuidados realizados pelo enfermeiro quando identifica uma situaçâo de violência contra o idoso.}

Verifica-se que os enfermeiros (as) citam várias alternativas para intervir no problema da violência contra o idoso, pois as condutas apresentadas, como acolhimento ao idoso, cuidados de enfermagem, acionar a equipe multidisciplinar, como o Serviço Social e de Psicologia, para comunicar ao Crea (Centro de Referência Especializado de Assistência Social), ao Conselho do Idoso, Secretaria de Direitos Humanos e Polícia. E, por fim, fazer as anotações (registro de enfermagem) no prontuário e preencher o SINAN (Sistema de Informação de Agravos de Notificação), mostram que os enfermeiros seguem um protocolo de conduta frente à violência contra a pessoa idosa, como se verifica na Figura 1.

O conhecimento e as estratégias adotadas, frente aos casos de violência, são importantes para a condução dos casos, e isso envolve a necessidade do profissional enfermeiro ter conhecimento abrangente a respeito da temática, com uma visão assistencial integral, interdisciplinar, holística, humanizada e contextualizada, 
Figura 1. Conduta frente à violência contra a pessoa idosa.

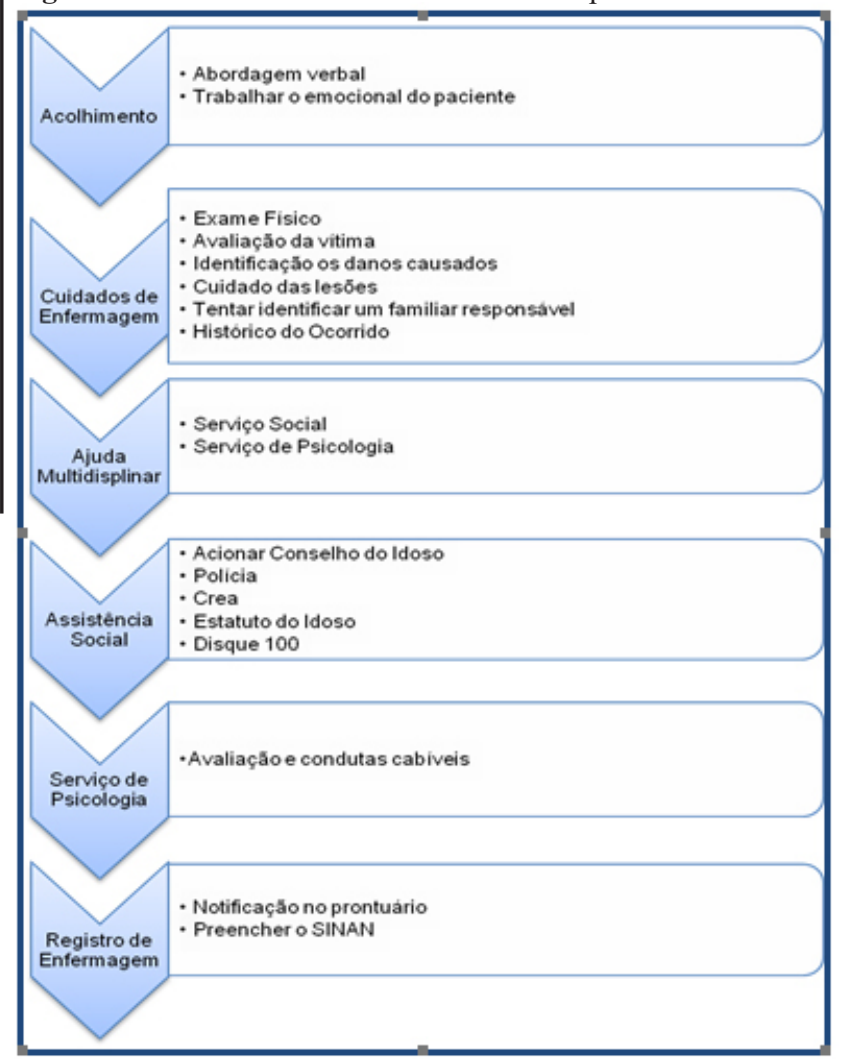

para que possa acolher, assistir e direcionar as pessoas em situação de violência. ${ }^{10: 222}$

O acolhimento é uma ação técnico- assistencial que tem o cliente como foco principal para a prestação de cuidados, provocando mu $\neg$ danças na relação profissional/cliente, melhoria na qualidade da assistência e reformulação no processo de trabalho da enfermagem. ${ }^{11}$

A fragilidade do idoso precisa ser trabalhada, através da empatia e respeito que o profissional de saúde, principalmente o (a) enfermeiro (a) precisa demonstrar ao atender o idoso maltratado e fragilizado, utilizando nesse momento sua intuição profissional, para conquistar e agilizar os trâmites legais que evitarão qualquer possibilidade de agressão futura, para isso, a interação entre família/idoso/enfermagem é fundamental no processo de descoberta dos problemas de maus tratos. 9:931

A Lei $n^{\circ} 12.461$, de 26 de julho de 2011 estabelece que os casos de suspeita ou confirmação de violência praticada contra idosos devem ser objeto de notificação compulsória pelos serviços de saúde públicos e privados à autoridade sanitária. A realização da notificação é obrigação dos profissionais da área de saúde em todas as esferas de atenção em casos suspeitos ou confirmados de violência devendo ser registrada no Sistema de
Informação de Agravos de Notificação (SINAN). Casos de violência sexual ou autoprovocada a notificação deve ser imediata, já os demais casos de violência que estejam confirmados, ou seja, suspeitas devem ser notificados no prazo máximo de uma semana. ${ }^{12}$

A rotina de notificação dos casos suspeitos ou confirmados de violência de qualquer natureza contra os idosos e a divulgação dos dados ajuda a dimensionar a questão da violência e ganhar mais visibilidade, pois o detalhamento do perfil da violência poderá aperfeiçoar as estratégias para alcançar os grupos mais vulneráveis e assim evitar novas ocorrências, bem como melhorar a orientação e tratamento às vítimas, além de nortear os enfermeiros a conduzir esses casos de violência. ${ }^{13}$

Observa-se nos depoimentos que uma das dificuldades em identificar o idoso vítima de violência está no fato de que nem sempre o próprio idoso tem consciência ou entende que certos comportamentos abusivos são violência. Também observa nos relatos que embora o idoso possa ter consciência de que está sento vítima de violência, ele omite o fato por medo de falar ou denunciar o agressor, especialmente porque é uma pessoa próxima, ou membro da família. ${ }^{14}$

Há dificuldade do enfermeiro em detectar a violência em idosos com capacidade limitada ou reduzida. A vergonha, a autocensura, a negação e o medo de represálias, bem como a preocupação em denunciar um membro da família, podem levar o idoso a resistir em denunciar o agressor às autoridades ou aos profissionais de saúde. Características culturais são barreiras que podem fazer com que alguns idosos se tornem menos propensos a denunciar possíveis abusos. Como também pode acontecer do idoso nem mesmo perceber que o que está vivenciando é abuso, ou se tiver conhecimento, simplesmente não sabe como proceder ou para quem denunciar. Isto faz com que $72 \%$ dos idosos que se dirigem às unidades de emergência após uma situação de violência, sequer faz menção ao fato. ${ }^{14}$

Uma das dificuldades em identificar o idoso vítima de violência está no fato de que nem sempre o próprio idoso tem consciência ou entende que certos comportamentos abusivos são violência. Também observa nos relatos que embora o idoso possa ter consciência de que está sento vítima de violência, ele omite o fato por medo de falar ou denunciar o agressor, especialmente porque é uma pessoa próxima, ou membro da família. ${ }^{14}$

\section{Conclusão}

O presente estudo se propôs verificar quais são as condutas adotadas pelo profissional enfermeiro ao identificar situações de violência e maus-tratos contra a pessoa idosa. 
Constatou-se que os enfermeiros têm um conhecimento abrangente sobre o conceito de violência, quando dizem se tratar de um fenômeno intencional no qual se faz uso da força, podendo se manifestar através de agressões físicas, verbal, psicológica, sexual, na qual também estão incluídos, a morte, as torturas, os conflitos e a fome, se constituindo em violação dos direitos humanos.

Ficou evidente que ao identificarem uma situação de violência contra a pessoa idosa, eles se valem de diversas estratégias de proteção ao idoso e, consequentemente de enfrentamento da violência. Uma vez que suas condutas estão pautadas no acolhimento, no trabalho em equipe, no aconselhamento e na denúncia aos órgãos competentes. Além disso, verificase que houve uma preocupação dos enfermeiros em relação ao registro nos prontuários e à notificação dos casos no SINAN. Este fato contribui para reduzir a subnotificação, além de contribuir para melhorar os dados epidemiológicos da violência contra os idosos.

Porém ficou evidente que diversos fatores intrínsecos ao idoso contribuem para dificultar a identificação da violência pelos enfermeiros, como medo, vergonha e omissão por medo do agressor ou por não querer denunciá-los por questões de afinidades parentais.

\section{Referências}

1. Paiva MM, Tavares DMS. Violência física e psicológica contra idosos: prevalência e fatores associados. Rev Bras Enferm. 2015 nov./ dez.;68(6):1035-41.

2. Silva CFS, Dias CMSB. Violência contra idosos na família: motivações, sentimentos e necessidades do agressor. Psicologia: Ciência e Profissão, 2016 jul./set.;36(3):637-52.

3. Souza RS, Bastos MAR. Acolhimento com classificação de risco: o processo vivenciado por profissional enfermeiro. REME - Revista Mineira de Enfermagem. 2008 out./dez.;12(4):581-86.

4. Mendonça AR, Durman S. O enfermeiro frente aos maus tratos contra o idoso: proposta de um protocolo. In: $3^{\mathrm{a}}$ Mostra de Trabalhos em Saúde Pública, UNIOESTE - Campos Cascavel - PR, 5 e 6 de novembro de 2009.

5. Brito AP. O papel do enfermeiro da unidade de estratégia de saúde da família no atendimento ao idoso vítima de violência. 2011. 34f. Monografia (Especialização em Saúde da Família) - Universidade Cândido Mendes, Governador Valadares - MG, 2011.

6. Bardin L. Análise de conteúdo. Lisboa: Edições 70, 1979.

7. Nomura FH, Gaidzinski RR. Rotatividade da equipe de enfermagem: estudo em hospital-escola. Rev Latino-am Enfermagem, 2005 set./ out.;13(5):648-53

8. Dahlberg LL, Krug EG. Violência: um problema global de saúde pública. Ciênc. Saúde Coletiva, 2006;11(supl):1163-78.

9. Camacho ACLF, Alves RR. Maus tratos contra os idosos na perspectiva da enfermagem: revisão integrative. Revenferm UFPE Recife, 2015 fev; 9 (supl):927-35.

10. Peralva TR, Araújo AKC, Bezerra CS et al. Violência doméstica na percepção de enfermeiros de serviço de emergência. ReonFacema, 2016 jul./ set.;2(3):221-28.
11. Costa PC, Garcia APRF, Toledo VP. Expectativa de enfermeiros brasileiros acerca do acolhimento realizado na atenção primária em saúde. Rev. Salud Pública. 2016;18(5):746-55.

12. Santos RC, Gonçalves RG, Ferreira LB. Indicadores de violência contra a pessoa idosa da Paraíba. In: Congresso Nacional Envelhecimento Humano, Maceió, 22 a 24 de novembro de 2017.

13. Santos JF, Silva ECT, Albuquerque TMO et al. Reflexões acerca da assistência de enfermagem no enfrentamento à violência contra idosos na atenção básica. In: Congresso Nacional Envelhecimento Humano, Maceió, 22 a 24 de novembro de 2017.

14. Stokowski LA. Forensic Issues for Nurses - Elder Abuse. Disponível em: <http:/www.medscape.org/viewarticle/578859>. Acesso em 23 nov. 2018 . 